der handelnden Vernunft und der Philosophie"! Die Kartographen präsentieren uns den Raum, die Welt, ihr Welt-Bild seit Jahrtausenden auf sehr unterschiedliche Art. Computertechnologie, Internet, Satelliten-gestützte Aufnahmetechniken revolutionieren gerade diese Weltaneignung. In solchen Zeiten des Umbruchs sind Kartographen besonders gefragt. Trotzdem soll das Institut für Kartographie der TU Dresden geschlossen werden.

Die Ausstrahlung kann auch über das Internet verfolgt werden.

Mark Vetter (Karlsruhe)

\section{- NIM-Tagung in Berlin}

\section{Tagungsbericht}

Mit dem Untertitel „Berichte aus der Medienwerkstatt" fand am 15. Januar 2015 der zweite Event zum Themengebiet "news-infographicsmaps" (http://news-infographicsmaps.net) statt, der in diesem Jahr als Kooperation mit der DGfK Sektion Berlin-Brandenburg von SND-DACH (Society for News Design Deutschland/Österreich/Schweiz) gestaltet wurde.

In dem einleitenden Beitrag stellten Katharina Stipp und Maria Thiele (KAMA Visual Statement, Berlin) eine Zusammenstellung der Historie der Landkarten in der Infografik vor. Andrew Timmins (Stern, Hamburg) berichtete unter dem Titel „Reibungspunkte zwischen Wort und Bild "zu sehr praxisbezogenen Vorstellungen und Wünschen und stellte diese in den operativen Kontext von Realität und Machbarkeit in der realen Arbeitsumgebung einer Redaktion. Thomas Heumann (FAZ, Frankfurt) gab mit seinem Beitrag zu „Kodifizierung - Fakten lesergerecht darstellen" einen sehr interessanten Einblick in die Usability-Diskussionen einer Zeitschriftenredaktion. Dabei weckten seine Diskussionen über nicht realisierte oder verworfene Alternativen der Darstellung die besondere Aufmerksamkeit der Zuhörer. Abschließend stellte Dr. Raimar
Heber (dpa-infografik, Berlin) unter seinem Thema „Barrierefreiheit Karten für Sehbehinderte und Blinde" eine Variante für diese Zielgruppe mit sogenannten taktilen Installationen vor.

Die Vortragenden brachten alle langjähriges Praxiswissen aus einem jeweils sehr bemerkenswerten und sehr erlebnisreichen Arbeitsumfeld in diese Veranstaltung. Die Beiträge fanden bei den Teilnehmern sehr hohes Interesse und die rege Diskussion hätte gern auch mehr als den Rahmen der verfügbaren Zeit ausgefüllt.

Das Interesse ist groß, die bei n-i-m 2015 angesprochenen besonderen Themenbereiche (Infografiken, Barrierefreiheit) zwischen den beteiligten Organisationen (DGfK und SND-DACH), mit den Referenten und mit den interessierten Teilnehmern weiter zu behandeln. Dies gilt insbesondere für die Anforderungen an den kurz- und mittelfristigen Bedarf von Entwicklungen, Testumgebungen und Usability-Untersuchungen. Ferner wäre es von Belang, Analysen und methodische Begründungen aus den Kognitionswissenschaften zu berücksichtigen. Denn es zeigte sich auf der Tagung, dass die situativ sehr unterschiedlichen Realisierungsbereiche der Geo-Visualisierung zu inhaltlich und organisatorisch unterschiedlichen Handlungsbedarf in nationalen und internationalen Fachkreisen führen.

Horst Kremers

DGfK Sektionsleiter

Berlin-Brandenburg

\section{Ravenstein-Förderpreis 2014}

\section{Die Preisträgerinnen und Preisträger und deren Werke}

Am 6. Oktober 2014 wurden im Friedenssaal der Stiftung Großes Waisenhaus in Potsdam die Förderpreise der KartographieStiftung Ravenstein verliehen. Damit fand die Preisverleihung nach der Veranstaltung 2013 im Sitzungssaal der Villa Mumm in Frankfurt am
Main erneut in einer hervorragenden Lokalität in festlichem Ambiente statt. Eine besondere Veranstaltung für die Nachwuchsförderung, die gerade in Brandenburg durch Ausbildungsinitiativen der Landesbehörde einen hohen Stellenwert hat. Besonders erfreulich war die sehr hohe Wettbewerbsbeteiligung mit 30 Teilnehmern aus 13 unterschiedlichen Einrichtungen, darunter zwei Doktoranden des Geographischen Instituts der Uni Heidelberg. So ein starkes Interesse war seit Jahren nicht mehr zu registrieren. Einerseits ist dies eine Folge der Bemühungen der Stiftung, den Ravenstein-Preis in den Focus der Fachöffentlichkeit zu bringen, andererseits auch Ausdruck des generell gestiegenen Interesses an der Kartographie und der Ausbildung. Man darf auf die Entwicklung im „International Map Year" gespannt sein und sich auf eine erneute Rekordbeteiligung für 2015 einstellen. Die Jury musste also aus beeindruckend vielen Facetten des Fachgebietes unterschiedliche Ansätze und Arbeiten vergleichen und Entscheidungen treffen.

Die Veranstaltung begann mit einer musikalischen Einstimmung Es folgten die Begrüßungsworte von Prof. Killiches, dem Präsidenten der LGB und Dr. Schöttler, dem Vorsitzenden der Kartographie-Stiftung Ravenstein, der die guten Beziehungen zur Mutterstiftung (Helga RavensteinStiftung) aufzeigte, die die attraktiven Geldpreise ermöglicht. Weiterhin galt der Dank den langjährigen Mitglie-

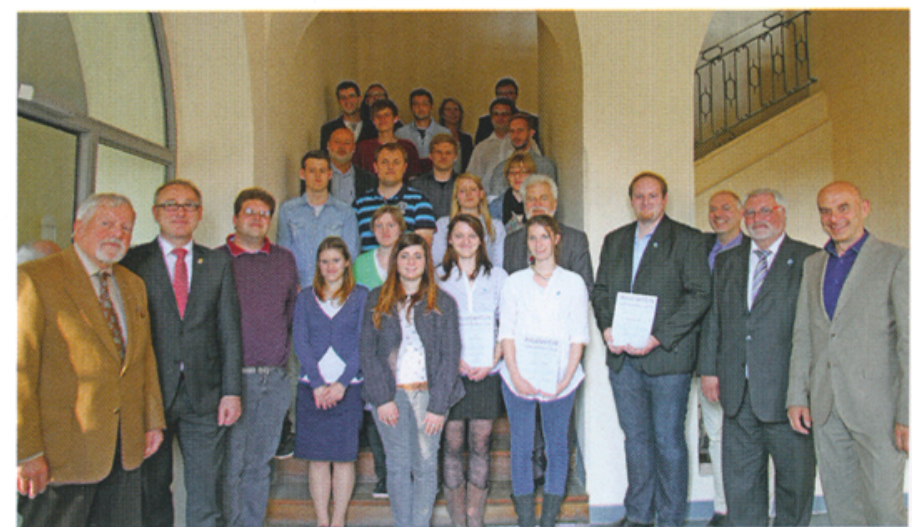

In der Mitte die Preisträger des Ravensteinförderpreises 2014. Am rechten und linken Bildrand die Juroren dern im Stiftungsvorstand und der Jury, Prof. Grünreich und Dipl. Geogr. Bodingbauer, die von der aktiven Mitarbeit zurücktraten und Platz für Nachfolger machen. Auch hier ist also die Kontinuität und Attraktivität, sich bei einem Förderpreis zu engagieren, gegeben.

Im Mittelpunkt der Veranstaltung standen natürlich die Preisträger! Vor viel Publikum, den stolzen Ausbildern, Firmenvertretern, Eltern und Fachvertretern, durften in feierlichem Ambiente die Arbeiten vorgestellt werden. Eine Ehre und gute Übung für die Teilnehmer, aber auch eine Herausforderung! Die Moderation übernahmen Dipl.-Geograph Dirk Zellmer und Dipl.-Ing. Wolfgang Kolb als Vertreter der Jury. Dabei differenziert die Jury zwischen den Arbeiten von Auszubildenden und von Studierenden. Insgesamt wurden bei den Auszubildenden 3 Preise und 3 Anerkennungen und bei den Studierenden 2 Preise und 3 Anerkennungen in einer Gesamthöhe von 4450,- Euro vergeben. Zusätzlich erhielten die Preisträger eine Jahresmitgliedschaft der DGfK, die Erstattung der Reisekosten zur Festveranstaltung und wurden durch eine Urkunde geehrt. Die Arbeiten spiegeln den hohen Stand der Ausbildung wieder.

Der 1. Platz ging an Vivian Hoen, Katharina Krüger, Sebastian Bein und Nicolas Wiggert von der Landesvermessung und Geobasisinformation Brandenburg. Sie habe eine wunderbare Arbeit über Kuriose

\begin{abstract}
r. Abschließend stelle Dr. Raimar
\end{abstract}

der Villa Mumm in Frankfurt am

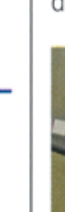

